\title{
Excessive thoracic computed tomographic scanning in sarcoidosis
}

\author{
Juan Maña, Alvin S Teirstein, David S Mendelson, Maria L Padilla, Louis R DePalo
}

Internal Medicine Service, Hospital de Bellvitge, Barcelona, Spain

J Maña

Division of Pulmonary and Critical Care Medicine

A S Teirstein

Department of Chest Radiology

D S Mendelson

Lung and Heart/Lung Transplantation, Pulmonary Section M L Padilla

Department of Medicine and Molecular Biology L R DePalo

The Mount Sinai Medical Center, New York 10029, USA

Reprint requests to: Dr A S Teirstein, Mount Sinai Medical Center, Division of Pulmonary and Critical Care Medicine, Box 1232, New York, New York 10029, USA.

Received 14 February 1995 Returned to authors Returned to

13 July 1995 Revised version

Accepted for publication 24 August 1995

\begin{abstract}
Background - The clinical value of computed tomographic (CT) scanning of the chest in the initial assessment of sarcoidosis was investigated.

Methods - One hundred consecutive patients referred to the sarcoidosis outpatient services of the Mount Sinai Medical Center, New York from 1990 to 1992 with a presumptive diagnosis of sarcoidosis were studied. The diagnosis was subsequently confirmed in all by a positive tissue biopsy sample or the KveimSiltzbach test. Clinical and laboratory data of each patient were reviewed. Chest radiographs were classified according to the classical stages of sarcoidosis. Thirty five of the 100 patients had a CT scan of the chest performed before presentation. The CT scans were compared with the presenting clinical data and standard chest radiographs in order to determine if they yielded useful additional information regarding diagnosis or treatment.
\end{abstract}

Results - The chest CT scan revealed no additional clinically relevant information compared with conventional chest radiographs in any of the 35 studies performed. In two patients mediastinal adenopathy was detected by CT scan which was not seen on standard radiographs. Two patients thought to exhibit hilar adenopathy and pulmonary infiltrations by standard radiography had no parenchymal disease on the CT scan. Bilateral parenchymal infiltrates were seen in one patient which were interpreted as unilateral infiltrates by standard radiographs. The variance between conventional radiographs and CT scans in these five patients was not clinically valuable.

Conclusions - CT scans of the chest do not add clinically useful information to the standard chest radiographs in the initial assessment of sarcoidosis in patients presenting with the typical standard radiological patterns. CT scanning of the thorax is indicated in patients with proven or suspected sarcoidosis when the standard chest radiographs are normal or not typical of sarcoidosis, when signs or symptoms of upper airway obstruction are present, when the patient has haemoptysis, if there is a suspicion of a complicating second intrathoracic disease, or the patient is a candidate for lung transplantation.

(Thorax 1995;50:1264-1266)

Keywords: sarcoidosis, computed tomographic (CT) scanning.
In the past decade there have been several reports promoting the value of computed tomographic (CT) scans of the chest in interstitial lung disease in general, ${ }^{1-4}$ and specifically in sarcoidosis. ${ }^{5-9}$ Studies have reported increased sensitivity for detection of mediastinal adenopathy and parenchymal infiltrations by CT scanning when compared with conventional radiographic techniques. Diagnostic patterns of abnormality on CT scans in sarcoidosis have been proposed. ${ }^{10}$ As a consequence, an increasing number of patients with presumed sarcoidosis initially present to the respiratory physician with a routine CT scan of the chest in addition to standard chest radiographs.

\section{Methods}

In order to assess the frequency of this practice and the contribution of the chest CT scan to the diagnosis and treatment of sarcoidosis, we analysed 100 consecutive patients with sarcoidosis who presented to the Division of Pulmonary and Critical Care Medicine at the Mount Sinai Medical Center, New York between 1990 and 1992.

The diagnosis of sarcoidosis was established by a positive tissue biopsy specimen or the Kveim-Siltzbach (KS) test in all patients. The age, sex, race, standard chest radiographic and CT scan patterns, and method of diagnosis were reviewed. The standard chest radiographs were classified according to the classical radiographic stages of sarcoidosis: stage I (hilar lymph node enlargement without pulmonary infiltrates), stage II (hilar lymph node enlargement accompanied by pulmonary infiltrates), stage III (pulmonary infiltrates alone), and stage IV (evidence of fibrosis). The clinical and radiographic data of the 35 patients presenting with CT scans were compared with those of the 65 without scans. All CT scans were reviewed to determine if they revealed intrathoracic lymph node enlargement, pulmonary infiltrates, or any other finding not seen on the standard chest radiographs. The CT scans were performed at various institutions and without a standard protocol. Only 13 were performed with high resolution technique. According to the referring physicians, the reason for requesting a CT scan in most instances was simply the presence of mediastinal adenopathy.

\section{Results}

The age, sex, race, chest radiographic stages, and biopsy procedures are shown in table 1 . The age range varied from 23 to 75 years in 
Table 1 Clinical data of 35 patients with sarcoidosis who underwent $C T$ scanning compared with 65 patients with sarcoidosis without CT scanning

\begin{tabular}{lcc} 
& $\begin{array}{c}\text { Patients with } \\
\text { chest CT } \\
(n=35)\end{array}$ & $\begin{array}{c}\text { Patients without } \\
\text { chest CT } \\
(n=65)\end{array}$ \\
\hline Age (range) & $23-75$ & $9-69$ \\
M:F & $14: 21$ & $25: 40$ \\
Race & $25(74 \%)$ & $42(65 \%)$ \\
White & $7(20 \%)$ & $19(29 \%)$ \\
Black & $1(3 \%)$ & $3(5 \%)$ \\
Puerto Rican & $1(3 \%)$ & $1(2 \%)$ \\
Indian & $1(3 \%)$ & $0(0 \%)$ \\
Chest radiographic stage & $14(40 \%)$ & $18(28 \%)$ \\
Stage 0 & $11(31 \%)$ & $31(48 \%)$ \\
Stage I & $7(20 \%)$ & $16(25 \%)$ \\
Stage II & $2(6 \%)$ & $0(0 \%)$ \\
Stage III & $12(34 \%)$ & $26(40 \%)$ \\
Stage IV & $12(34 \%)$ & $20(31 \%)$ \\
Diagnostic biopsy procedures* & $1(3 \%)$ & $2(3 \%)$ \\
Kveim-Siltzbach test & $2(14 \%)$ & $5(8 \%)$ \\
Bronchial biopsy & $4(11 \%)$ & $4(6 \%)$ \\
Open lung & $1(3 \%)$ & $1(2 \%)$ \\
Mediastinal lymph node & $2(6 \%)$ & $7(25 \%)$ \\
Peripheral lymph node & $1(3 \%)$ & \\
Scalene lymph node & (3kin & \\
Upper respiratory tract & &
\end{tabular}

* Several patients had more than one diagnostic procedure.

the CT group and from 9 to 69 years in the non-CT group; most patients were between the ages of 20 and 40 years. There was no difference between the groups in sex and ethnicity. A slightly higher percentage of patients in the CT group had stage I radiographs, while in the non-CT group there was a higher percentage of patients with stage II and III radiographs. The two patients with stage IV radiographs had CT scans. The diagnosis was established by the Kveim-Siltzbach test, flexible bronchoscopic biopsy, or mediastinoscopy in approximately $80 \%$ of both groups, with diagnosis by extrathoracic biopsy in the remainder.

In 30 of the 35 patients who underwent CT scanning no new information was obtained when compared with their standard chest radiographs (table 2). CT scans revealed mediastinal lymph node enlargement not appreciated by the chest radiographs in two patients with stage III disease. These two were classified as stage II by CT scan. One patient with a stage II standard radiograph which revealed unilateral parenchymal infiltrates and hilar adenopathy had bilateral infiltrates on the CT scan. Two patients whose standard chest radiograph was interpreted as stage II had hilar adenopathy but no demonstrable parenchymal disease (that is, stage I) on the CT scans. The information contributed by CT scan in these five patients was considered to be of no clinical significance. The single patient with a stage 0 standard radiograph had no adenopathy or

Table 2 Comparison between staging by CT scanning of the chest and chest radiography in 35 patients with sarcoidosis

\begin{tabular}{lcc}
\hline & $\begin{array}{l}\text { Standard chest } \\
\text { radiograph }\end{array}$ & $\begin{array}{l}\text { CT scan } \\
\text { of thorax }\end{array}$ \\
\hline Stage 0 & 1 & 1 \\
Stage I & 14 & $16^{*}$ \\
Stage II & 11 & 11 \\
Stage III & 7 & $5 \dagger$ \\
Stage IV & 2 & 2 \\
\hline
\end{tabular}

* CT scanning demonstrated no lung infiltrates in two patients deemed stage II disease by standard chest radiography.

†CT scanning demonstrated mediastinal lymph nodes in two patients with stage III disease by standard chest radiography. parenchymal lesions on the CT scan. However, her CT scan of the neck confirmed the clinical impression of laryngeal sarcoidosis.

\section{Discussion}

In 1973 Winterbauer et al proposed that the finding of a typical stage I chest radiograph in an asymptomatic young patient was diagnostic of sarcoidosis. ${ }^{11}$ Indeed, the radiographic patterns of sarcoidosis are readily identified and should lead the physician to a logical sequence of studies to confirm that diagnosis. However, increasing numbers of studies have indicated a greater sensitivity of CT scanning compared with conventional radiography in elucidating the subtleties of the many patterns of diseases whih afflict the pulmonary parenchyma. For example, patients with Pneumocystis carinii pneumonia and normal standard chest radiographs often exhibit pulmonary opacities on a CT scan. ${ }^{12}$ Honeycombing, pleural disease, mediastinal lymph node enlargement, and delineation of nodules from reticular shadows are appreciated better by CT scan than standard chest radiographs. ${ }^{5910}$ Thus, in many patients with diffuse lung diseases the CT scan is a valuable aid to diagnosis. In addition, the results of at least one study suggest that the addition of high resolution CT scanning increases the concurrence of the differential diagnosis. ${ }^{13}$ This enthusiasm for CT scans has influenced physicians confronted with a patient with probable sarcoidosis. At the first visit, usually with a conventional chest radiograph exhibiting typical hilar and mediastinal adenopathy, with or without pulmonary opacities, a CT scan of the thorax is frequently requested. In the current study 34 of the 35 CT scans were so requested.

There is little question that CT scanning, particularly high resolution CT scanning, is more sensitive than conventional radiography in elucidating the intrathoracic anatomical changes in diffuse lung diseases. ${ }^{14}$ In our study CT scanning generally provided a more detailed and presumably more complete assessment of nodal and parenchymal disease than conventional radiography. In sarcoidosis CT scans have demonstrated nodular opacities along the bronchovascular bundles, interlobular septae, major fissures, and substernal regions. ${ }^{7} \mathrm{CT}$ scanning also reveals small pleural effusions, ${ }^{56}$ bullae, and mediastinal lymph node enlargement not detected by conventional radiographs. ${ }^{69}$ Some studies suggest that CT scanning has a very high sensitivity for the diagnosis of sarcoidosis. However, Müller ${ }^{15}$ has stated that "the CT findings are not pathognomonic". Some studies have proposed that high resolution CT scanning parallels pulmonary dysfunction ${ }^{16}$ and, because of superior detection of irreversible fibrosis and bullae, is of prognostic value in sarcoidosis. ${ }^{17-19}$ However, other investigators have questioned the clinical relevance of prognostic superiority of CT scanning over standard chest radiographs which have served this purpose in the past. ${ }^{20}$ Finally, it has been proposed that thoracic CT scans are an aid to the choice of bronchoscopic biopsy site. $^{6}$ 
It is rarely necessary to perform a CT scan for diagnostic purposes in sarcoidosis. No pattern of CT abnormality has been identified that has been generally accepted as a substitute for pulmonary function, serological testing, gallium-67 scanning, and/or clinical assessment as guides to the treatment of pulmonary sarcoidosis. In 34 of our patients who had a CT scan the diagnosis was strongly suggested by the clinical picture and conventional chest radiographs. Eleven referring physicians stated that they assumed a CT scan was indicated in patients with mediastinal abnormalities and/or diffuse lung disease seen on conventional chest radiographs. In a single patient with previously diagnosed extrathoracic sarcoidosis and normal lungs and mediastinum by standard chest radiographs, upper airway narrowing was shown on the CT scan. The course and treatment of this patient alone was influenced by the results of the CT scan.

Although studies of low dose CT scans are in progress, the current standard CT scan exposes patients to $0 \cdot 04-0 \cdot 14 \mathrm{~Gy}^{21}{ }^{22}$ High resolution CT scanning delivers a significantly greater dose of radiation. The minimal information obtained, the additional radiation, and the extra financial burden place a major responsibility on the treating physician to have precise indications for ordering a CT scan of the thorax in a patient presenting with a typical standard chest radiograph of sarcoidosis.

This study is not meant to preclude the use of CT scans in all patients with sarcoidosis. We propose the following indications for thoracic high resolution CT scanning of the thorax in patients undergoing study for suspected or proven sarcoidosis: (1) normal standard chest radiograph; (2) standard chest radiograph atypical for sarcoidosis; (3) haemoptysis in a patient with proven sarcoidosis; (4) suspicion of a second complicating disease (for example, pulmonary neoplasm, infection or pleural process); and (5) candidates for lung transplantation.

We are indebted to Roz Richardson and Shirley Palleja for their invaluable aid in preparing this manuscript. Dr J Maña was a invaluable aid in preparing this manuscript. Dr J Maña was a Investigación Sanitaria, Ministerio de Sanidad y Consumo",
Madrid, Spain. Dr A S Teirstein was supported by the Catherine and Henry Gaisman Foundation, New York.

1 Bergin CO, Müller NL. CT in the diagnosis of interstitial lung disease. $A \mathcal{R} R$ 1985;145:505-10.

2 Müller NL, Miller RR. Computed tomography of chronic diffuse infiltrative lung disease. Part 1. Am Rev Respir Dis 1990;142:1206-15.

3 Müller NL, Miller RR. Computed tomography of chronic diffuse infiltrative lung disease. Part 2. Am Rev Respir Dis 1990;142:1440-8.

4 Hansell DM, Kerr IH. The role of high resolution computed tomography in the diagnosis of interstitial lung disease. Thorax 1991;46:77-84.

5 Solomon A, Kreel L, McNicol M, Johnson N. Computed tomography in pulmonary sarcoidosis. 7 Comput Assist Tomogr 1979;3:754-8.

6 Hamper UM, Fishman EK, Khouri NF, Johns CJ, Wang KP, Siegelman SS. Typical and atypical CT manifestations of pulmonary sarcoidosis. $\mathcal{F}$ Comput Assist Tomogr 1986; of pulmonary

7 Brauner MW, Grenier P, Mompoint D, Lenoir S, Oremoux $\mathrm{H}$. Pulmonary sarcoidosis: evaluation with high resolution CT. Radiology 1989;172:467-71.

8 Müller NL, Kullnig P, Miller RR. The CT findings of pulmonary sarcoidosis: analysis of 25 patients. $A \mathscr{F}$ 1989; 162:1179-82.

9 Sider L, Horton ES. Hilar and mediastinal adenopathy in sarcoidosis as detected by computed tomography. F Thorac Imaging 1990;5:77-80.

10 Lynch DA, Webb WR, Gamsu G, Stulbarg M, Golden J. Computed tomography in pulmonary sarcoidosis. $7 \mathrm{Com}$ Computed tomography in pulmon
put Asst Togr 1989;13:405-10.

11 Winterbauer RH, Belie M, Moores KD. A clinical interpretation of bilateral hilar adenopathy. Ann Intern Med 1973;78:65-71.

12 Moskovic E, Miller R, Pearson M. High resolution computed tomography of Pneumocystis carinii pneumonia in AIDS. Clin Radiol 1990;42:239-43.

13 Grenier P, Valeyre D, Cluzel P, Brauner MW, Lenoir S, Chastang C. Chronic diffuse interstitial lung disease: diagnostic value of chest radiography and high-resolution CT. Radiology 1991;179:123-32.

14 Nakata H, Kimoto T, Nakayama T, Kido M, Miyazaki N, Harada S. Diffuse peripheral lung disease: evaluation by high resolution computed tomography. Radiology 1985; high resolutic

15 Müller NL. Differential diagnosis of chronic diffuse infiltrative lung disease on high-resolution computed tomography. Semin Roentgenol 1991;26:132-42.

16 Bergin CJ, Bell DY, Coblentz CL, Chiles C, Gamsu G, MacIntyre NR, et al. Sarcoidosis: correlation of pulmonary parenchymal pattern at CT with results of pulmonary function tests. Radiology 1989;17:619-24.

17 Müller NL, Mawson JB, Mathieson JR, Abboud R, Ostrow DN, Champion P. Sarcoidosis: correlation of extent of disease at CT with clinical, functional and radiographic disease at CT with clinical, functional

18 Murdoch J, Müller NL. Pulmonary sarcoidosis: changes on follow-up CT examination. AfR 1992;159:473-7.

19 Brauner MW, Lenoir S, Grenier P, Cluzel P, Battesti JP, Valeyre D. Pulmonary sarcoidosis: CT assessment of lesion reversibility. Radiology 1992;182:349-54.

20 Austin JHM. Pulmonary sarcoidosis: what are we learning from CT? Radiology 1989;171:603-4.

21 Naidich DP, Marshall CH, Gribbin C, Arams RS, McCauley DI. Low-dose CT of the lungs: preliminary observations. Radiology 1990;175:729-31.

22 Zwirewich CV, Mayo JR, Müller NL. Low-dose highresolution CT of lung parenchyma. Radiology 1991; 180:413-7. 